04

\title{
Влияние низкочастотных флуктуаций магнитного поля на удержание плазмы в торсатроне Ураган-3М при редких частотах столкновений
}

\author{
(C) В.К. Пашнев, Э.Л. Сороковой, А.А. Петрушеня, Ф.И. Ожерельев \\ Национальный научный центр „Харьковский физико-технический институт“, \\ Институт фризики плазмы, \\ 61108 Харьков, Украина \\ ฯ e-mail: sorokovoy@ipp.kharkov.ua
}

(Поступило в Редакцию 5 марта 2018 г.)

В торсатроне Ураган-3М в условиях ВЧ-создания и нагрева плазмы (частоты, близкие к ионноциклотронной) при наличии бутстреп-тока с помощью диамагнитных измерений проведено измерение энергосодержания плазмы. Рассмотрен баланс мощности при быстром нагреве плазмы и на его основе проведена оценка ВЧ-мощности, поглощаемой плазмой в объеме удержания. Рассчитано поведение потерь энергии в течение разряда и проведено обсуждение влияния флуктуаций магнитного поля в диапазоне частот $0.5-70 \mathrm{kHz}$ на потери энергии.

DOI: 10.21883/JTF.2019.01.46962.103-18

\section{Введение}

В торсатроне Ураган-3М (У-3М) [1] для создания и нагрева плазмы традиционно используется ВЧ-нагрев на частотах, близких к ионной циклотронной [2]. В одном из режимов удается получить низкоплотную достаточно горячую плазму [3], в которой наблюдается продольный ток $[3,4]$ относительно большой величины. Такой режим характеризуется редкими частотами столкновений между частицами плазмы (банановая область на кривой Галеева-Сагдеева).

Исследования поведения плазмы в режиме редких столкновений представляет интерес не только для понимания удержания плазмы в данной конкретной установке, но может быть интересно и с общефизической точки зрения, так как будущий термоядерный реактор на основе тороидальной ловушки будет работать именно в режиме редких столкновений.

Определение параметров плазмы в этом режиме на торсатроне У-3М сопряжено с большими трудностями, в частности, определение температуры плазмы. Это связано, прежде всего, с искажением функции распределения в условиях редких столкновений. Поэтому для решения ряда задач оказывается полезным использование диамагнитных измерений, которые регистрируют общую запасенную энергию в плазменном объеме. Такие измерения полезны, например, при анализе баланса мощности.

В тороидальных установках с внешним вращательным преобразованием, каким является торсатрон У-3М, при наличии относительно большого продольного тока для использования диамагнитного эффекта в целях диагностики плазмы необходимо знание распределения тока и угла вращательного преобразования по сечению плазменного шнура. Определение распределения указанных выше параметров само по себе является слож- ной задачей. В настоящей работе приведены условия для достаточно точного определения энергосодержания плазмы на основе методики диамагнитных измерений в стеллараторных системах.

ВЧ-нагрев при относительно высоком уровне поглощаемой плазмой мощности относится к быстрым методам нагрева. Быстрый нагрев - нагрев, при котором время изменения уровня вводимой в плазму мощности существенно меньше времени изменения параметров плазмы, которые, в свою очередь, значительно меньше скинового времени проникновения магнитного поля плазменных токов в плазму и элементы металлического окружения. Как показано в работах [5-7], в случае быстрого нагрева в балансе мощности предложено также учитывать мощность, идущую на изменение магнитного поля в области удержания плазмы. Причем, мощность, идущая на нагрев плазмы, согласно работе [7], может составлять только 1/3 от вводимой мощности при быстром нагреве.

Одной из причин превышения потерь тепла и частиц из плазменного объема в тороидальных магнитных ловушках относительно предсказаний неоклассической теории является возбуждение в плазме различных неустойчивостей. В частности, к таким неустойчивостям относятся различные ветки альфвеновских, геодезических и дрейфовых волн, характерной особенностью которых являются флуктуации магнитного поля. Для регистрации флуктуаций магнитного поля на торсатроне У-ЗМ в одном из малых сечений установлен набор магнитных датчиков.

Целью настоящей работы является определение энергосодержания плазмы в режиме нагрева плазмы с редкими частотами столкновений, оценка уровня поглощаемой плазмой ВЧ-мощности, потерь энергии из плазменного объема и связи регистрируемых флуктуаций магнитного поля с этими потерями. 

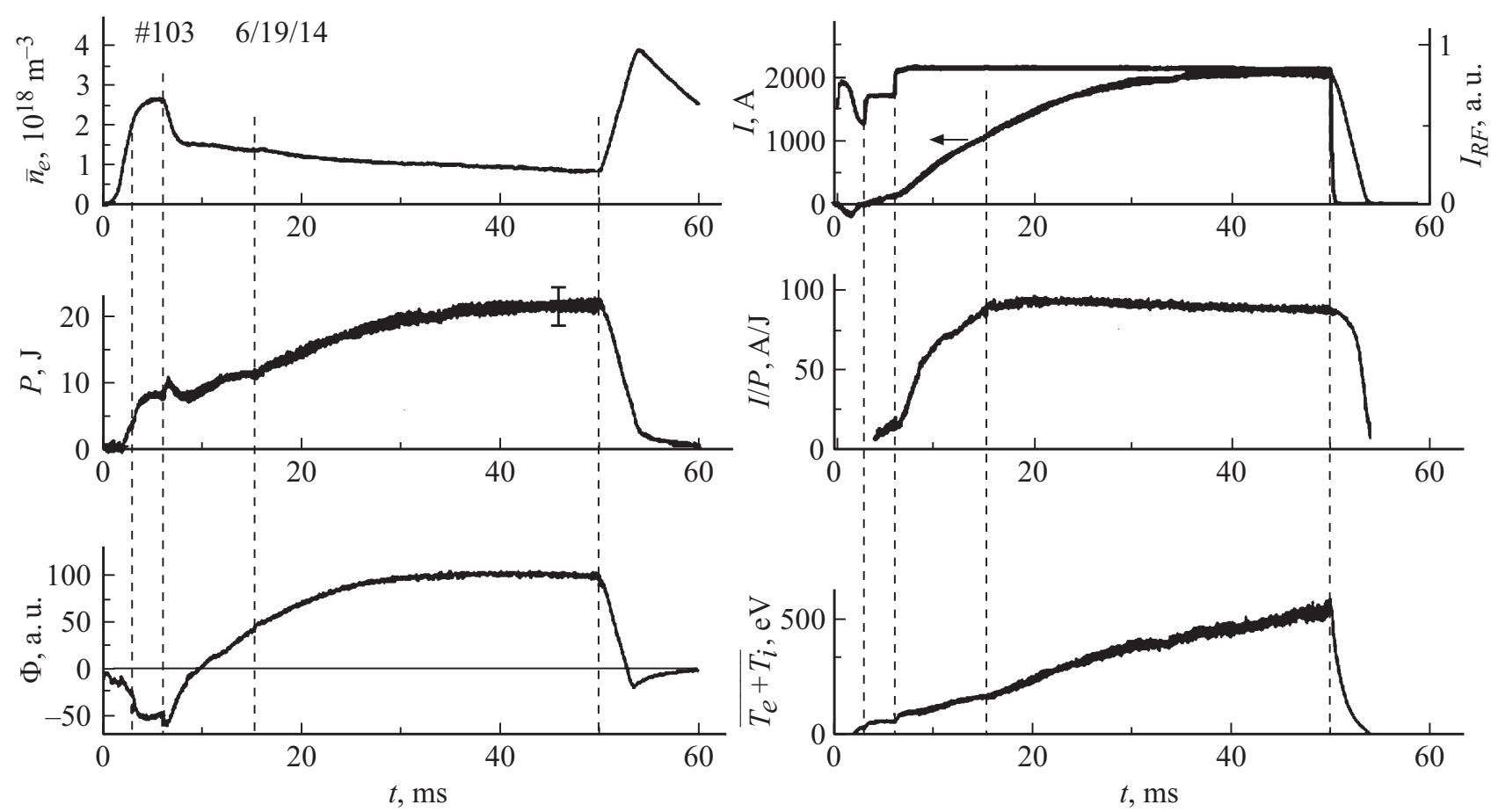

Рис. 1. Временно́е поведение основных параметров плазмы. $\bar{n}_{e}-$ средняя плотность плазмы; $P$ - энергосодержание плазменного шнура; $\Phi$ - изменение тороидального магнитного потока; $I-$ продольный плазменный ток; $I_{R F}-$ ток в ВЧ-антенне; $I / P$ - отношение величины тока в плазме к ее энергосодержанию; $\overline{T_{e}+T_{i}}-$ сумма средних температур электронов и ионов, определенные из диамагнитных измерений. Штриховыми линиями обозначены моменты включения дополнительной мощности и ее выключения, а также момент особенности в поведении энергосодержания плазмы.

\section{Условия эксперимента и результаты исследований}

Эксперименты проводились на торсатроне У-ЗМ в режиме ВЧ-нагрева на частоте $\omega=2 \pi f \approx 0.8 \omega_{\mathrm{Bi}}(0)$, где $\omega_{\mathrm{Bi}}(0)-$ ионная циклотронная частота на геометрической оси тора. В данном эксперименте частота ВЧ-генератора составляла $f \approx 8.6 \mathrm{MHz}$. Номинальное напряжение на генераторной лампе составляло $9 \mathrm{kV}$. Магнитное поле на геометрической оси было $B \approx 0.7 \mathrm{~T}$.

У-3М представляет собой трех заходный торсатрон с большим радиусом плазменного шнура $R \approx 1 \mathrm{~m}$, средним радиусом последней магнитной поверхности $a \approx 0.1 \mathrm{~m}$. Винтовая обмотка помещена в вакуумную камеру объемом около $70 \mathrm{~m}^{3}$. Распределение угла вращательного преобразования может быть описано следующим выражением:

$$
\iota_{s t}=\iota_{0}+\iota_{2}(r / a)^{2}
$$

где $\iota_{0} \approx 0.22, \iota_{2} \approx 0.11$ и $r-$ средний текущий радиус магнитной поверхности. В качестве рабочего газа использовался водород, который подавался в проточном режиме. Пробой рабочего газа осуществлялся при давлении около $10^{-5}$ Torr при анодном напряжении генераторной лампы существенно ниже номинального.

На рис. 1 приведено временное поведение основных параметров плазменного разряда. Плотность плазмы измерялась $2 \mathrm{~mm}$ интерферометром. Энергосодержание плазменного шнура определялось с помощью диамагнитных измерений. Диамагнитная петля представляет собой две коаксиальные круговые катушки, диаметры которых различаются на $0.01 \mathrm{~m}$. Идея измерения состоит в том, что полезный сигнал $\Phi$ для обеих катушек одинаков, а сигналы, связанные с протеканием токов в металлическом окружении, пропорциональны разности площадей катушек. Максимальная частота регистрируемых диамагнитной петлей сигналов составляет $20 \mathrm{kHz}$.

Выражение, связывающее энергосодержание плазменного шнура с другими измеряемыми параметрами разряда, имеет вид

$$
P=-\frac{3}{4} R B \Phi+\frac{3}{2} \pi R \frac{I^{2}}{c^{2}}+3 \pi^{2} \frac{B}{c} \int_{0}^{a} j \frac{\partial}{\partial r}\left(r^{2} \int_{0}^{r} \iota_{s t} x d x\right) d r .
$$

Здесь $P=\frac{3}{2} \int p d V-$ энергосодержание плазменного шнура, $p=n_{e} k\left(T_{e}+T_{i}\right)$ - давление плазмы, $V-$ объем, $T_{e}$ - температура электронов, $T_{i} \approx \sum_{j} \alpha_{j} T_{j}, T_{j}$ температура ионов сорта $j$ и $\alpha_{j}-$ их относительная концентрация, $I=2 \pi \int_{0}^{a} j r d r-$ продольный плазменный ток, измеряемый поясом Роговского и $j-$ его плотность. 
Выражение (2) представляет собой хорошо известное соотношение, полученное для некрутых тороидальных систем с $a / R \ll 1$, в котором два первых слагаемых в правой части используются в токамаках (смотри, например, $[8,9])$, а третье слагаемое - учитывает наличие внешнего вращательного преобразования [10]. В случае отсутствия продольного тока изменение тороидального магнитного потока $\Phi$ происходит за счет диамагнитных токов, текущих поперек магнитного поля. При наличии продольного тока квадратичный член по току обеспечивает парамагнитное изменение магнитного потока. В установках с внешним вращательным преобразованием знак магнитного потока меняется в зависимости от направления тока.

Как видно из рис. 1, в начальной стадии разряда пока ток мал в изменении тороидального магнитного потока превалирует диамагнитная составляющая. С ростом продольного тока знак сигнала меняется на противоположный. Это указывает на тот факт, что направление тока совпадает с направлением магнитного поля, т. е. ток увеличивает вращательное преобразование.

Как видно из выражения (2), изменение тороидального магнитного потока при данной величине энергосодержания плазмы и достаточно сильном продольном токе зависит от распределения $\iota_{s t}(r)$ и $j(r)$. Это сильно усложняет применение диамагнитных измерений в стеллараторных системах. Однако при малых значениях величины

$$
\beta=\frac{8 \pi}{B^{2}} p \ll \beta_{p}=\frac{1}{2} \iota_{s t}^{2}(a) \frac{a}{R}
$$

изменение стеллараторного угла вращательного преобразования в процессе разряда незначительно. В нашем эксперименте $\beta \leq 4 \cdot 10^{-4}$, что существенно меньше $\beta_{p} \sim 10^{-2}$. Поэтому в расчетах энергосодержания плазменного столба при диамагнитных измерениях следует учитывать изменение во времени только распределения тока, а распределение $\iota_{s t}(r)$ использовать в виде (1). Проведенные расчеты показали, что зависимость величины $P$ от распределения продольного тока не сильно критична. Если представить распределение тока в виде

$$
j=j_{0}(r / a)^{m}\left[1-r^{n} / a^{n}\right]^{g}+j_{1},
$$

то в случае $j_{1}=0$ выражение (2) можно представить в виде

$$
P=-\frac{3}{4} R B \Phi+\frac{3}{2} \pi R \frac{I^{2}}{c^{2}}+2 \pi B \iota_{s t}(a) \iota \frac{l}{c} .
$$

В этом случае для реальных распределений тока $(n=1-3)$ и параметров магнитной системы У-3М в случае $m=0, g=2$, величина $l$ будет равна $0.27 \pm 0.03$ (распределение с максимумом тока вблизи магнитной оси); для $m=0, g=0$ величина $l$ будет равна 0.4 (однородное распределение) и при $m \neq 0 ; g=2$, $l=0.43 \pm 0.05$ (распределение тока с $j(0)=0$ ).
Таким образом, видно, что в экспериментах на торсатроне У-3М при диамагнитных измерениях продольный магнитный поток больше зависит от величины продольного тока $I$, чем от его распределения по радиусу. Однако для более точного определения энергосодержания плазменного шнура полезным будет знание природы тока и, как следствие, его распределение.

Для данного эксперимента в плазме возможны такие токи: бутстреп-ток [9], ток увлечения [11] и ток, возникающий при быстром нагреве [10].

Бутстреп-ток возникает в тороидальной плазме в режиме редких столкновений между частицами плазмы. Выражение для плотности этого тока в токамаке имеет вид [9]

$$
j_{b}=-1.46 c \sqrt{\frac{R}{r}} \frac{1}{B \iota} \frac{\partial p}{\partial r} .
$$

Как видно из этого выражения, плотность бутстрептока вблизи магнитной оси стремится к нулю, а наибольшее значение реализуется в области максимального градиента давления плазмы.

Ток увлечения $I_{g}$ может возникнуть в результате взаимодействия электронов плазмы с ВЧ-полем, обеспечивающим нагрев плазмы при наличии асимметрии распространения электромагнитных волн вдоль тора. Выражения для этого тока можно представить в виде

$$
I_{g}=k W .
$$

где $W$ - вводимая в плазму ВЧ-мощность и $k-$ некий коэффициент пропорциональности. Антенна, используемая для ВЧ-нагрева плазмы в торсатроне У-3М, не предназначена для возбуждения бегущих в одном направлении электромагнитных волн. Эффективность возбуждения такого тока на частотах, близких к ионной циклотронной без создания специальных антенн, не велика. К тому же, в условиях редких частот столкновений между частицами плазмы, наличие запертых частиц резко уменьшает, эффективность возбуждения токов увлечивается на частотах, близких к ионной циклотронной [12].

Пространственное распределение тока увлечения зависит от области поглощения ВЧ-мощности, поэтому локализация такого тока может меняться в течение разряда. В наших условиях направление тока увлечения неизвестно и зависит от многих факторов (направление магнитного поля, направление и скорость полоидального и тороидального вращений плазмы и т.д.).

Ток, возникающий при быстром нагреве, является следствием изменения полоидального магнитного потока из-за смещения магнитных поверхностей при быстром изменении $\beta$ в условиях вмороженнности магнитного поля в плазму. Величина этого тока пропорциональна $\beta^{2}$. Ток имеет максимальное значение на фазе роста и спада $\beta$ и равен нулю на стационарной стадии разряда. Оценки показывают, что максимальное значение тока, возникающего при быстром нагреве, не превышает 50 А для наших значений параметров плазмы 
и магнитной системы, поэтому этот ток не рассматривается в качестве основного, на фоне регистрируемого тока величиной $2 \mathrm{kA}$.

Рассмотрим имеющиеся экспериментальные данные. В результате измерения смещения продольного тока было показано [13], что в начальной стадии разряда смещение тока в горизонтальном направлении наружу составляет около $4 \mathrm{~cm}$, а к концу разряда - около $1 \mathrm{~cm}$. Этот факт можно объяснить тем, что в начале разряда ток локализован вблизи магнитной оси, смещенной относительно геометрического центра наружу на $5 \mathrm{~cm}$. К концу разряда основная часть тока сосредоточена вблизи наружной поверхности, смещенной на величину около $1 \mathrm{~cm}$ наружу.

Величина продольного тока близка к величине тока, рассчитанного, согласно выражению (5), и зависит от направления магнитного поля [4], что совпадает с зависимостью, представленной в выражении (5).

Как видно из рис. 1 , величина $I / P \approx$ const устанавливается после $15 \mathrm{~ms}$, что указывает на зависимость величины продольного тока от давления плазмы. Из выражения (5) видно, что плотность бутстреп-тока пропорциональна $\partial p / \partial r$. Если представить распределение давления в виде $p=p_{0}\left[1-(r / a)^{d}\right]$, то можно получить согласно выражению (5):

$$
\frac{I}{P}=\frac{0.97}{\pi a^{2}} \frac{1}{B\left(\iota_{0}+0.45 \iota_{2}\right)} \sqrt{\frac{a}{R}} \frac{d+2}{d+1 / 2} .
$$

Согласно рис. 1, значение величины $I / P$ практически не меняется начиная с $15 \mathrm{~ms}$. С этого момента величина $I / P$, полученная в эксперименте, совпадает с выражением (7) для значений бустреп-тока в токамаках при значениях $d \approx 2.4-2.8$. Однако наблюдаемый профиль плотности [14] указывает на более пикированный профиль давления (т.е. $d \leq 2$ ). В этом случае для использования формулы (5) для торсатрона У-3М следует уменьшить коэффициент при плотности тока на 20-30\%.

Рост величины $I / P$ до $15 \mathrm{~ms}$ можно объяснить расширением области редких столкновений в процессе повышения температуры плазмы.

Приведенные экспериментальные данные не дают однозначного ответа на природу наблюдаемого продольного тока, поэтому были проведены эксперименты со ступенчатым увеличением вводимой в плазму мощности [3]. Очевидно, что ток увлечения в этом случае должен сразу нарастать с постоянной времени $L / \Omega$, где $L-$ индуктивность продольного тока, $\Omega-$ его сопротивление. Бустреп-ток в первый момент времени не должен изменяться и лишь впоследствии возрастает до величины, соответствующей установившемуся давлению. В этом эксперименте ток в момент ступенчатого увеличения мощности не изменился.

Таким образом, можно утверждать, что в нашем разряде наблюдается только бутстреп-ток.

Параметры разряда таковы, что появление бутстрептока вполне обосновано.
Согласно неоклассической теории [9], частицы находятся в области редких столкновений, если выполняется условие

$$
v_{j} \ll \frac{v_{T j^{i}}}{R} \varepsilon^{3 / 2},
$$

где $v_{j}$ - частота столкновений частицы сорта $j, v_{T j}-$ их тепловая скорость и $\varepsilon=\frac{r}{R}$.

Из условия (8) следует, что для плотности $n_{e} \approx 10^{18} \mathrm{~m}^{-3}$ и $Z=1$ условиями редких столкновений являются $T_{e} \gg 130 \mathrm{eV}$ и $T_{i} \gg 100 \mathrm{eV}$. По аналогии с работой [15], где обсуждалось временное поведение основных параметров разряда аналогичного исследуемому, считаем, что при номинальном уровне вводимой в плазму мощности средние по сечению температуры электронов и ионов изменяются в диапазоне $T_{e}=100-300 \mathrm{eV}$ и $T_{i}=80-200 \mathrm{eV}$, а величина среднего заряда ионов приближается к $Z=1$ к концу разряда. Такие параметры разряда позволяют считать, что в значительной области сечения разряда реализуется режим редких столкновений. В таком разряде должен наблюдаться бутстреп-ток.

Для бутстреп-тока, $j(0)=0 \quad$ и $l=0.43$ (выражение $(4))$, кроме того, полагаем, что $j_{1}=0$, ток увеличения и ток быстрого нагрева малы. Учитывая вышесказанное, на основании выражения (4) было рассчитано значение величины энергосодержания плазмы, приведенное на рис. 1.

Высокочастотная мощность наращивается ступенчато в 3 этапа (рис. 1,2): на $1.8 \mathrm{~ms}$ - происходит пробой, на 3 и $6 \mathrm{~ms} \mathrm{ВЧ-мощность,} \mathrm{подаваемая} \mathrm{в} \mathrm{плазму,} \mathrm{увеличи-}$ вается. После $3 \mathrm{~ms}$, когда вводится относительно малая мощность, идет быстрый рост энергосодержания и плотности плазмы. Процесс быстрого роста параметров плазмы длится около $1 \mathrm{~ms}$. Температура плазмы при этом достигает $100 \mathrm{eV}$. Затем начинается насыщение параметров плазмы. После $6 \mathrm{~ms}$ сначала происходит быстрый рост энергосодержания плазмы приблизительно на $30 \%$ за $0.5 \mathrm{~ms}$, а затем - быстрый $(2 \mathrm{~ms})$ спад к начальным значениям энергосодержания плазмы и далее - рост энергосодержания в течение всего разряда. Включение дополнительной ВЧ-мощности на $6 \mathrm{~ms}$ приводит также к быстрому уменьшению плотности, ускорению роста тока и повышению температуры плазмы. В дальнейшем, наблюдается постоянный медленный спад плотности и рост температуры до конца разряда. С 9-й до приблизительно 30-й ms от начала разряда наблюдается постоянный рост энергосодержания плазмы, а начиная c 38-й ms энергосодержание плазмы практически не меняется.

Проведенные оценки скинового времени $\tau_{s k}=\frac{4 \pi \sigma a^{2}}{c^{2}}$, где $\sigma$ - проводимость, показывает, что при наших параметрах плазмы скиновое время изменяется в диапазоне $\tau_{s k} \approx 10 \mathrm{~ms}$ на начальном этапе разряда до $\tau_{s k} \approx 40 \mathrm{~ms}$ к концу разряда. Из рис. 1,2 видно, что в начале разряда характерное время изменения энергосодержания плазмы составляет $\tau_{f} \approx 1 \mathrm{~ms}$ при величине роста и падения фронта ВЧ-импульса менее $50 \mu \mathrm{s}$, а в середине разряда $\tau_{f} \approx 10 \mathrm{~ms}$, что заметно меньше $\tau_{s k}$ для всей 
длительности разряда. Считаем, что плазма вморожена в магнитное поле, и для описания процесса нагрева можно использовать теорию, разработанную для быстрого нагрева $[5,6]$.

Баланс мощности при быстром нагреве плазмы, согласно работе [6], можно представить в виде

$$
\begin{aligned}
\frac{d}{d t} \int\left(\frac{3}{2} p+\frac{B^{2}}{8 \pi}\right) d V+\oint & \left(\frac{5}{2} p \mathbf{v}+\mathbf{q}+\frac{c}{4 \pi}[\mathbf{E B}]\right) d \mathbf{S}=W^{*} \\
\delta \mathbf{E}= & \frac{1}{c}[\mathbf{u B}] \\
\frac{d}{d t} \int\left(\frac{3}{2} p+\frac{B^{2}}{8 \pi}\right) d V & =\frac{\partial}{\partial t} \int\left(\frac{3}{2} p+\frac{B^{2}}{8 \pi}\right) d V \\
& +\oint\left(\frac{3}{2} p+\frac{B^{2}}{8 \pi}\right) \mathbf{u} d \mathbf{s}
\end{aligned}
$$

Здесь $\mathbf{v}=\mathbf{v}_{0}+\mathbf{u}-$ скорость движения плазмы, $\mathbf{u}-$ скорость движения плазмы при быстром нагреве, $\mathbf{v}_{0}$ скорость движения плазмы в стационарных условиях. $\mathbf{E}=\mathbf{E}_{0}+\delta \mathbf{E}-$ электрическое поле, $\delta \mathbf{E}-$ поле, возникающее при быстром нагреве, $\mathbf{E}_{0}$ - электрическое поле в стационарах условиях, $\mathbf{q}$ - поток тепла из плазменного объема, $W^{*}$ - мощность, идущая на нагрев плазмы, изменение магнитного поля и потери, не связанные с элементарными процессами (конвективные процессы, теплопроводность и дифузия), $\mathbf{S}$ - площадь поверхности. Выражение (10) описывает условие вмороженности плазмы в магнитное поле.

Будем считать, что полная мощность, поглощаемая плазмой в объеме удержания

$$
W=W^{*}+W_{B},
$$

где $W_{B}$ - мощность, идущая на элементарные процессы (ионизация, диссоциация, перезарядка, излучение и т.д.). Кроме того:

$$
\begin{gathered}
\frac{\partial}{\partial t} \int \frac{B_{v}^{2}+B_{r}^{2}}{8 \pi} d V=\frac{\partial}{\partial t} L \frac{l^{2}}{2}, \\
\frac{c}{4 \pi}[\delta \mathbf{E B}]=\mathbf{u} \frac{B^{2}}{4 \pi}, \\
\tau_{E}^{*}=\frac{P}{\oint\left(\frac{5}{2} p \mathbf{v}_{0}+\mathbf{q}+\frac{c}{4 \pi}\left[\mathbf{E}_{0} \mathbf{B}\right]\right) d \mathbf{S}+W_{0}} .
\end{gathered}
$$

Здесь $B_{v}$ и $B_{r}-$ компоненты магнитного поля в квазицилиндрической системе координат $\mathbf{r}, \boldsymbol{\vartheta}, \boldsymbol{\phi}$, где $\mathbf{r}$ - компонента направлена по малому радиусу тора, $\vartheta$ - полоидальная компонента по малому обходу тора, $\phi$ - компонента по большому обходу тора и $\tau_{E}^{*}-$ временной масштаб, характеризующий потери энергии из плазменного объема. Величина $\tau_{E}^{*}-$ совпадает с известной величиной энергетического времени жизни $\tau_{E}$ на стационарной стадии разряда. На динамичиских участках может наблюдаться существенное различие, связанное с определением величины $\tau_{E}$.
Если учесть, что, согласно (10):

$$
u_{r}=-\frac{1}{2 \pi a B} \frac{\partial \Phi}{\partial t}-\frac{c \iota a}{2 \pi R^{2} B} L \frac{\partial I}{\partial t},
$$

то, следуя работе [6], выражение (9) можно переписать в виде

$$
\frac{\partial P}{\partial t}-\frac{3}{4} B R \frac{\partial \Phi}{\partial t}+\left(I-\frac{3}{4} \frac{c \iota a^{2} B}{R}\right) L \frac{\partial I}{\partial t}+\frac{P}{\tau_{E}^{*}}=W .
$$

Здесь $\iota=\iota_{s t}(a)+\iota_{\tau}(a)-$ полный угол вращательного преобразования на границе плазмы, $\iota_{\tau}(a)=\frac{2 I R}{c a^{2}} B-$ угол вращательного преобразования, создаваемый продольным плазменным током.

Выражение (17) представим в виде

$$
\frac{\partial P}{\partial t}+\frac{P}{\tau_{E}^{*}}=W+\frac{3}{4} B R \frac{\partial \Phi}{\partial t}+\left(\frac{3}{4} \frac{c \iota a^{2} B}{R}-I\right) L \frac{\partial}{\partial t} I .
$$

Тогда правая часть выражения (18) представляет собой мощность, идущую на нагрев плазмы. В стадии нагрева, если тороидальный поток $\Phi$ изменяется в диамагнитную сторону, мощность, связанная с изменением этого магнитного потока, отбирается от вводимой мощности. Если $\Phi$ изменяется в парамагнитную сторону, то мощность прибавляется. Аналогичная ситуация существует при изменении магнитного потока плазменного тока I. При выключении нагрева все изменяется с точностью до наоборот.

Обозначим первые три слагаемых в левой части выражения (17) как $W_{n}$

$$
W_{n}=\frac{\partial P}{\partial t}-\frac{3}{4} B R \frac{\partial \Phi}{\partial t}+\left(I-\frac{3}{4} \frac{c \iota a^{2} B}{R}\right) L \frac{\partial I}{\partial t},
$$

которая представляет собой мощность, идущую на нагрев плазмы и изменение энергии магнитного поля. В этом случае поглощаемая плазмой мощность будет равна

$$
W_{n}+\frac{P}{\tau_{E}^{*}}=W
$$

На рис. 2 приведено временное поведение $W$ - мощности поглощаемой плазмой, энергосодержание плазменного объема $P$, флуктуации полоидальной компоненты магнитного поля $\tilde{B}_{v}$, зарегистрированное одним из магнитных зондов и расположенного на радиусе $b=16.8 \mathrm{~cm}$ вне объема удержания в диапазоне частот $1.5-70 \mathrm{kHz}$ и величины $W_{n}$.

Величина $W_{n}$, которая представляет собой мощность, идущую на нагрев плазмы и изменение энергии магнитного поля (см. выражение (19)), имеет сложное временное поведение. Положительные значения величины $W_{n}$ соответствуют мощности, идущей на увеличение энергии плазмы и магнитного поля, а отрицательные на их уменьшение при быстром нагреве. В начальной стадии разряда наблюдается резкий пик $W_{n}$, достигающий величины $9.3 \mathrm{~kW}$, который после включения первой 

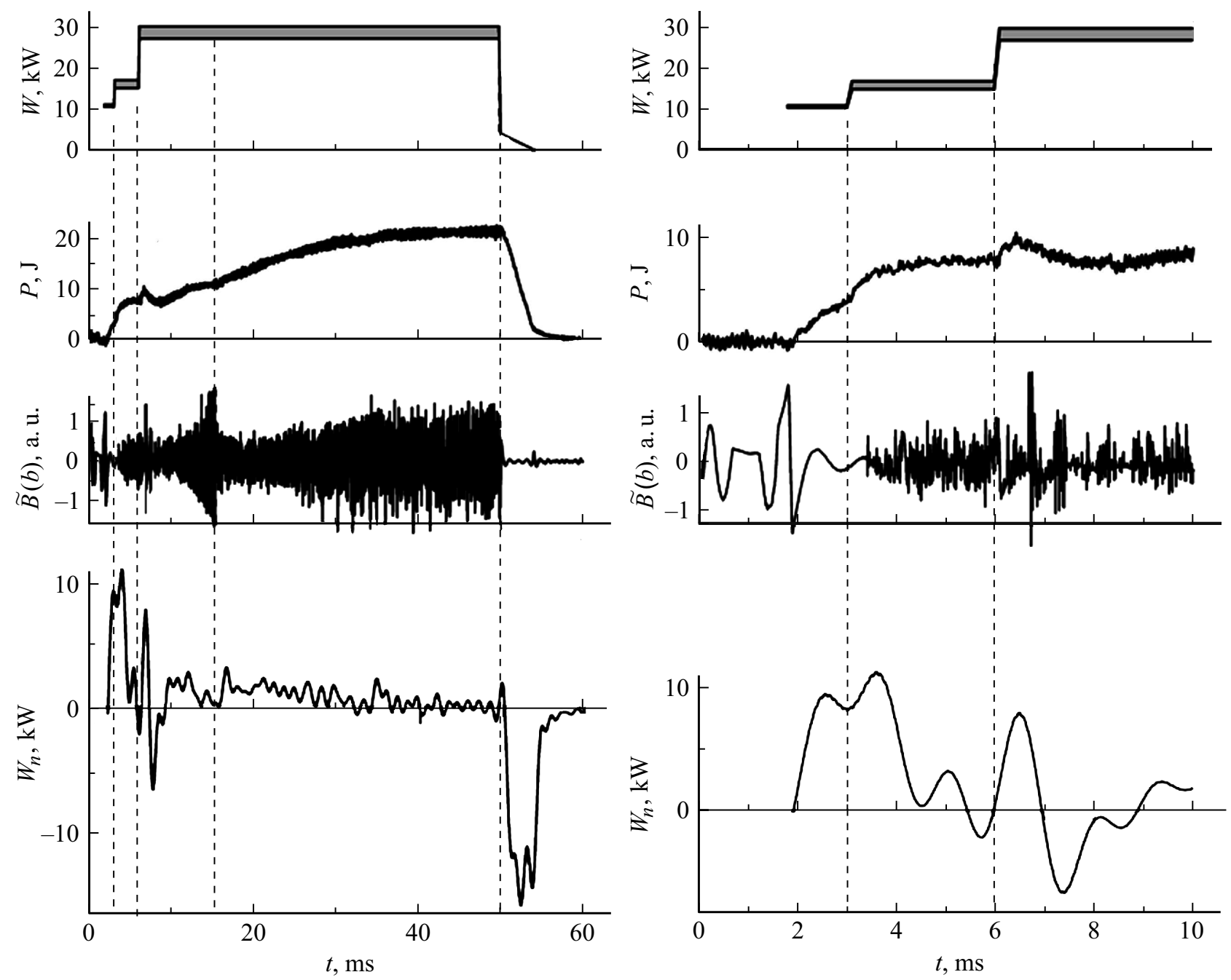

Рис. 2. Временно́е поведение мощности, поглощаемой плазмой $W$, энергосодержания плазмы $P$, флуктуаций магнитного поля $\tilde{B}(b)$ в диапазоне $1.5-70 \mathrm{kHz}$ и мощности, идущей на нагрев плазмы и изменение энергии магнитного поля, $W_{n}$, для двух масштабов времени.

ступени дополнительной мощности на 3-й ms достигает значения $11 \mathrm{~kW}$. Затем идет спад величины $W_{n}$ практически до нуля. Это происходит в связи с тем, что $\frac{\partial P}{\partial t} \rightarrow 0$, а продольный ток становится положительным и начинает возврастать. После включения второй ступени дополнительной мощности на $6 \mathrm{~ms}$ наблюдается короткий положительный пик до $7.7 \mathrm{~kW}$, совпадающий с пиком на энергосодержании плазмы, а затем мощный отрицательный пик до $-6.5 \mathrm{~kW}$, коррелирующий с уменьшением энергосодержания плазмы, спадом средней плотности плазмы и резким ростом продольного тока (см. рис. 1). В дальнейшем до конца ВЧ-нагрева величина $W_{n}$ изменяется в районе нуля. Это указывает на то, что основная мощность идет на компенсацию потерь энергии из плазмы. После выключения ВЧ-нагрева величина $W_{n}$ отрицательная, так как идет уменьшение энергии, запасенной в плазме и магнитном поле.

В начальной стадии разряда в момент включения ВЧ-мощности наблюдаются периоды времени с линей- ным ростом энергосодержания плазмы. Используя это, можно оценить величину $\tau_{E}^{*}$. Изменением тока можно пренебречь и выражение (20) можно переписать в виде

$$
2.5 \frac{\partial P}{\partial t}+\frac{P}{\tau_{E}^{*}}=W .
$$

Из выражения (21) видно, что при быстром нагреве, когда плазма вморожена в магнитное поле, и в отсутствие продольного тока, мощность, идущая на нагрев плазмы и изменение магнитного поля, $W_{n}=2.5 \frac{\partial P}{\partial t}$. То есть мощность, затрачиваемая на изменение магнитного поля, в 1.5 раза больше идущей на нагрев плазмы.

При постоянных значениях величин $\tau_{E}^{*}$ и $W$ решение уравнения (21) известно

$$
P=P_{0}\left[1-\exp \left(-t / 25 \tau_{E}^{*}\right)\right] .
$$

В случае $t \ll 2.5 \tau_{E}^{*}$ выражение $(21)$ приводится к виду

$$
P=P_{0} \frac{t}{2.5 \tau_{E}^{*}} .
$$


Выражение (23) описывает временное поведение энергосодержания плазмы в начальной стадии разряда на линейных участках роста энергосодержания плазмы.

Имеющиеся экспериментальные данные позволяют провести оценку потери энергии и уровня поглощаемой плазмой ВЧ-мощности в объеме удержания. На начальной стадии разряда величина $\tau_{E}^{*}$ не может быть меньше $1.7 \mathrm{~ms}$, чтобы выполнялось условие $2.5 \tau_{E}^{*} \gg \tau_{E}=0.85 \mathrm{~ms}$. Оценка мощности, идущей на протекание элементарных процессов (ионизация нейтрального газа, перезарядка и неоклассический перенос), составляет около $1.2 \mathrm{~kW}$. Поэтому, если считать, что потери плазмы описываются неоклассической теорией, то максимальное значение величины $\tau_{E}^{*} \leq 3 \mathrm{~ms}$. С учетом вышесказанного, согласно выражению (19), поглощаемая ВЧ-мощность в начальной стадии разряда может находиться в диапазоне $W=10.5-11.6 \mathrm{~kW}$.

Для оценки поглощаемой плазмой мощности $W_{n}$ после ее первого ступенчатого увеличения берем прирост после включения первой ступени $\Delta W_{n}=1.9 \mathrm{~kW}, P=7.5 \mathrm{~J}$ и минимальное $\tau_{E}^{*}=1.1 \mathrm{~ms}$, вычисленное по линейному росту энергосодержания $P$ после ступенчатого роста мощности за $3 \mathrm{~ms}$. Получаем величину $17 \mathrm{~kW}$ (ионизация + перезарядка + перенос - $4.6 \mathrm{~kW})$.

Аналогично мощность, поглощаемая плазмой после второго ступенчатого увеличения, равна $W \approx 27-30 \mathrm{~kW}$ на интервале 46-50 ms. Этот результат не противоречит данным болометрических измерений, выполненных ранее для аналогичного разряда, которые дали значение излучаемой плазмой мощности на уровне $12-14 \mathrm{~kW}$ [16]. Ток в антенне после включения второй ступени мощности практически не меняется, поэтому мы считаем, что мощность, поглощаемая плазмой, постоянная.

На рис. 2 приведено временное поведение флуктуаций магнитного поля, регистрируемое одним из 15 магнитных датчиков, установленных в одном из сечений вдали от антенны. Зонды с соответствующей электронной аппаратурой позволяли регистрировать флуктуации магнитного поля в плазменном объеме на уровне $10^{-7} \mathrm{~T}$ в полосе частот до $70 \mathrm{kHz}$. Частотный диапазон ограничивался скоростью оцифровки сигнала и длиной подводящих кабелей.

Флуктуации магнитного поля появляются приблизительно через $1.5 \mathrm{~ms}$ после пробоя. Появление флуктуаций совпадает с уменьшением скорости роста плотности плазмы и ее энергосодержания (см. рис. 1 и 2). То есть в плазменном объеме должны быть достигнуты определенные параметры (может быть величина $\beta$ ) для возбуждения колебаний. Сразу после выключения ВЧ-нагрева флуктуации пропадают. Временное поведение $\tilde{B}$ является довольно сложным. Например, начиная c $13 \mathrm{~ms}$ после начала разряда, амплитуда флуктуаций возрастает. Рост амплитуды колебаний совпадает с замедлением скорости роста энергосодержания. На $15 \mathrm{~ms}$ (момент обозначен пунктирной линией) в течение $10 \mathrm{~ms}$ амплитуда флуктуаций уменьшается в несколько раз,

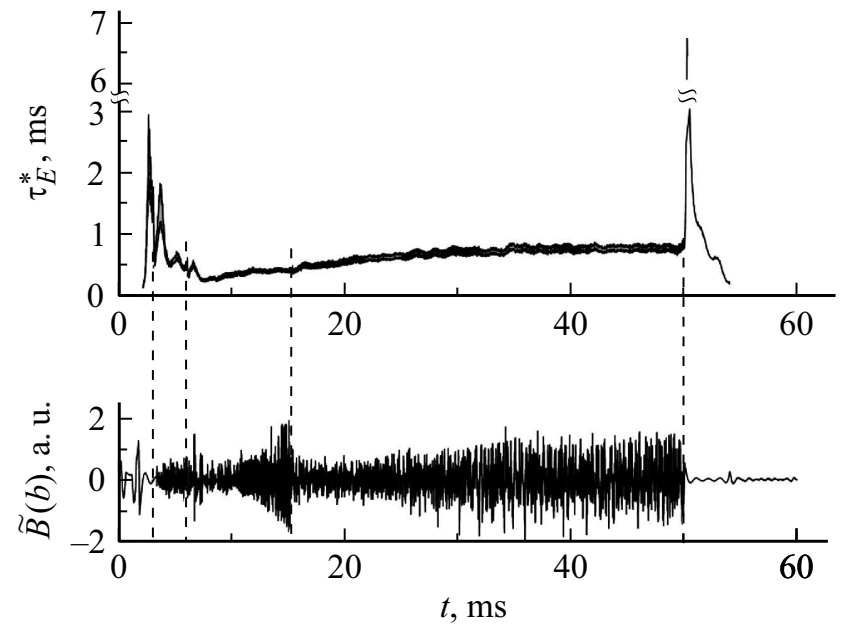

Рис. 3. Поведение величины $\tau_{E}^{*}$ и флуктуаций магнитного поля в течение разряда.

и с этого момента резко возрастает скорость роста энергосодержания плазмы. Кстати, в этот же момент прекращается рост величины $I / P$ (см. рис. 1).

На рис. 3 приведено поведение величины $\tau_{E}^{*}$ в течение ВЧ-разряда. Для этого использовалось выражение

$$
\tau_{E}^{*}=\frac{P}{W-W_{n}} .
$$

Хочется отметить, что в начале разряда $\tau_{E}^{*} \approx 1.7-3 \mathrm{~ms}$, а сразу же после выключения ВЧ-нагрева $\approx 6.7 \mathrm{~ms}$, при этом величина флуктуаций магнитного поля ниже чувствительности регистрирующей системы. В течение всего остального разряда $\tau_{E}^{*}$ изменяется от $\quad 0.25-0.35 \mathrm{~ms}$ около $7.5 \mathrm{~ms}$ после подачи ВЧимпульса (через $1.5 \mathrm{~ms}$ после резкого увеличения ВЧ-мощности) до $0.7-0.8 \mathrm{~ms}$ к концу разряда. С 7й по $27 \mathrm{~ms}$ наблюдается почти линейный рост $\tau_{E}^{*}$ за исключением диапазона $13.5-16.5 \mathrm{~ms}$. В районе $13.5-16.5 \mathrm{~ms}$ амплитуда флуктуаций меняется в разы, a энергетическое время жизни изменяется на $20 \%$ с $\tau_{E}^{*}=0.38-0.43$ до $0.43-0.52 \mathrm{~ms}$. Катастрофа, связанная с резким падением удержания плазмы, которая произошла в районе 3.5-й ms, коррелирует с появлением флуктуаций магнитного поля большой амплитуды.

Как видно из рис. 3 , линейная связь между амплитудой флуктуаций магнитного поля и поведением $\tau_{E}^{*}$ отсутствует, однако отчетливо прослеживается качественная связь этих параметров.

Причин отсутствия прямой связи между поведениями потерь энергии и амплитудой регистрируемых колебаний магнитного поля может быть несколько. Во-первых, за основные потери могут быть ответственны колебания в другом частотном диапазоне. Во-вторых, флуктуации магнитного поля возбуждаются в глубине плазменного объема и по какой-то причине не могут распространяться к границе и излучаться в окружающее пространство. 


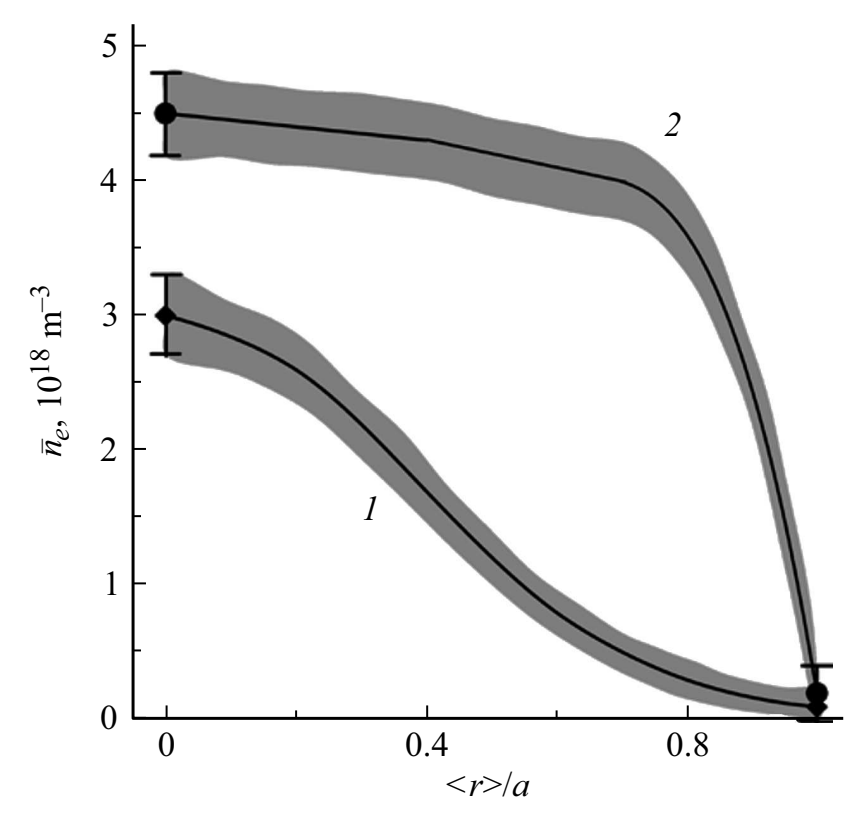

Pис. 4. Профиль плотности плазмы в различные моменты времени в зависимости от среднего радиуса магнитной поверхности $\langle r\rangle$, нормированного на средний радиус последней магнитной поверхности: $1-$ во время ВЧ-нагрева $(t \approx 15 \mathrm{~ms})$; 2 - после выключения ВЧ-нагрева $(t \approx 50.5 \mathrm{~ms})$.

И, в-третьих, не только флуктуации магнитного поля ответственны за потери энергии из плазменного объема. Потери также могут быть обусловлены разными механизмами, а вот резкое увеличение $\tau_{E}^{*}$, которое происходит на $15 \mathrm{~ms}$ и интерпретируется как переход в режим улучшенного удержания [16-18], по-видимому, полностью определятся наблюдаемыми флуктуациями магнитного поля. Следует отметить, что ошибка в определении величины вводимой в плазму мощности практически не сказывается на картине поведения $\tau_{E}^{*}$ в течение разряда.

Изменение $\tau_{E}^{*}$ отражается на профиле плотности плазмы (рис. 4). Для определения профиля плотности использовались два измеренных значения по отсечке прохождения через плазменный объем СВЧ-волн различной частоты [14] и ленгмюровскими зондами на границе [19], при этом профиль плотности между двумя этими точками должен соответствовать интегралу по хорде, измеренному $2 \mathrm{~mm}$ интерферометром. На рис. 4 возможный диапазон изменения плотности обозначен цветом.

Из рис. 4 видно, что на активной стадии разряда профиль плотности имеет острую форму. Максимальный градиент находится на среднем радиусе магнитной поверхности $\langle r\rangle / a \sim 0.4$, а минимальный градиент в области $\langle r\rangle / a>0.8$. По-видимому, именно на границе плазменного шнура происходят события, обеспечивающие потери из плазменного объема.

После выключения ВЧ-нагрева профиль плотности становится плоским с крутым градиентом на границе. Такой профиль может быть обеспечен рядом причин.
Прежде всего, как видно из рис. 2, резким уменьшением уровня флуктуаций $\tilde{B}$ после выключения ВЧ-нагрева. Кроме того, уменьшение продольного тока вызывает появление напряжения на обходе тора на уровне $U=\frac{\partial}{\partial t} L I \approx 3 \mathrm{~V}$. В этом случае при редких частотах столкновений, согласно неоклассической теории, должно происходить пинчевание плазмы [9]. Этот вопрос обсуждался ранее в работе [3]. Очевидно, эти две причины должны привести к заметному улучшению удержания плазмы, что и наблюдается в эксперименте (величина $\tau_{E}^{*}$ достигает значений почти $7 \mathrm{~ms}$ ).

Приведенные экспериментальные данные не позволяют утверждать, что наблюдается прямая связь между уровнем вводимой в плазму ВЧ-мощности и потерями из плазменного объема. С нашей точки зрения, для объяснения имеющихся данных больше всего подходит теория „канонического“ профиля [20,21]. Действительно, в начальной стадии ВЧ-разряда, когда поглощаемая ВЧ-мощность мала, профиль энергосодержания плазмы, по-видимому, не сильно отличается от „канонического“ для данной магнитной конфигурации. Возрастание уровня энергсодержания приводит к изменению профиля и его заметному отличию от „канонического“. Это вызывает возбуждение неустойчивости и уменьшение величины $\tau_{E}^{*}$. Резкое увеличение вводимой в плазму мощности в первый момент приводит к увеличению энергосодержаения, а далее к его уменьшению, что может быть следствием заметного искажения профиля $P$ и увеличению потерь. Далее в процессе разряда профиль $P$ приближается к „каноническому“, что приводит к увеличению $\tau_{E}^{*}$ в течение разряда. После выключения ВЧ-нагрева профиль становится „каноническим“ с учетом пинчевания. При этом сразу пропадают флуктуации магнитного поля $\tilde{B}$ и происходит резкое улучшение удержания.

\section{Выводы}

1. Показана возможность использования диамагнитных измерений для определения энергосодержания плазмы в стеллараторных системах с продольным током при малых значениях величины $\beta$ и известной природе тока.

2. Подтверждена неоклассическая природа продольного тока (бутстреп-ток), возникающего в исследуемом разряде.

3. Рассмотрен баланс мощности при быстром нагреве плазмы и на его основе проведена оценка ВЧ-мощности, поглощаемой плазмой в объеме удержания.

4. Рассчитано поведение величины $\tau_{E}^{*}$, характеризующей потери из плазмы, в течение разряда. Показано, что появление в процессе разряда флуктуаций магнитного поля резко уменьшает величину $\tau_{E}^{*}$.

5. Показано, что поведение колебаний магнитного поля в диапазоне частот $0.5-70 \mathrm{kHz}$ качественно объясняет изменение потерь тепла из объема удержания в течение исследуемого ВЧ-разряда. 
В заключении авторы благодарят М.М. Козулю за предоставленные данные об излучаемой антенной ВЧ-мощности в течение разряда, Р.О. Павличенко за любезно предоставленную информацию о средней плотности плазмы, а также экипажу установки У-3М за обеспечение проведения эксперимента.

\section{Список литературы}

[1] Besedin N.T., Bykov V.E., Georgiyevskiy A.V. et al. // Probl. Atom. Sci. Tech. 1987. Vol. 4. P. 7.

[2] Chechkin V.V., Grigor'eva L.I., Pavlichenko R.O. et al. // Plasma Phys. Reps. 2014. Vol. 40. N 8. P. 601.

[3] Pashnev V.K., Sorokovoy E.L. // Probl. Atom. Sci. Tech. 2008. Vol. 14. N 6. P. 31-33.

[4] Гутарев Ю.В., Назаров Н.И., Павличенко О.С., Паинев В.К. и др. // Письма в ЖЭТФ. 1987. Т. 46. Вып. 2. C. $60-62$.

[5] Danilkin I.S. // Plasma Phys. Rep. 1998. Vol. 24. N 9. P. 796800.

[6] Pustovitov V.D. // Plasma Phys. Rep. 2011. Vol. 37. N 2. P. 109-117.

[7] Andreev V.F., Dnestrovskij Yu.N., Ossipenko M.V. et al. // Plasma Phys. Contr. F. 2004. Vol. 46. P. 319-335. [Kantor M.Yu., Bertschinger G., Bohm P. et al. // Proc. of the 36th EPS Conf. on Plasma Phys. Sofia, 2009. Vol. 33E. P-1.184.]

[8] Мирнов С.В. Физические процессы в плазме токамака. М.: Энергоатомиздат, 1983. С. 44.

[9] Галеев А.А., Сагдеев Р.3. Вопросы теории плазмы. М.: Атомиздат, 1973. Т. 7. С. 205, 210, 238.

[10] Пустовойтов В.Д., Шафранов В.Д. // Вопросы теории плазмы. М.: Атомиздат, 1987. Т. 15. С. 146, 248, 256.

[11] Klima R. // Plasma Phys. 1973. Vol. 15. P. 1031; Fisch N.J. // Phys. Rev. Lett. 1978. Vol. 41. N 13. P. 843.

[12] Cordey J.G., Eldington T., Start D.F.H. // Plasma Phys. 1982. Vol. 24. P. 73.

[13] Pashnev V.K., Tarasov I.K., Sitnikov D.A. et al. // Probl. Atom. Sci. Tech. 2013. Vol. 1. P. 15.

[14] Pashnev V.K., Sorokovoy E.L., Petrushenya A.A. et al. // Probl. Atom. Sci. Tech. 2015. Vol. 1. P. 290.

[15] Pashnev V.K., Sorokovoy E.L., Petrushenya A.A. et al. // Probl. Atom. Sci. Tech. 2010. Vol. 6. P. 24.

[16] Pashnev V.K., Sorokovoy E.L., Berezhnyj V.L. et al. // Probl. Atom. Sci. Tech. 2010. Vol. 16. N 6. P. 17-20.

[17] Volkov E.D., Adamov I.Yu., Arsen'ev A.V. et al. // Proc. of the 14th IAEA Conf. on Plasma Physics and Controlled Nuclear Fusion Research. Würzburg, Germany, 1992. Vienna: IAEA. 1993. Vol. 2. P. 679-688.

[18] Chechkin V.V., Grigor'eva L.I., Sorokovoy Ye.L. et al. // Plasma Phys. Rep. 2009. Vol. 35. N 10. P. 852.

[19] Kasilov A.A., Grigor'eva L.I., Chechkin V.V. et al. // Probl. Atom. Sci. Tech. 2015. Vol. 1. P. 24.

[20] Razumova K.A., Andreev V.F., Eliseev L.G. et al. // Nucl. Fusion. 2011. Vol. 51. 083024.

[21] Dnestrovskij Yu.N. Self-Organization of Hot Plasmas. Berlin: Springer, 2014. 134 p. 